\title{
Archipel
}

ARCHIPEL Études interdisciplinaires sur le monde insulindien

$98 \mid 2019$

Varia

\section{Singapura before Raffles: Archaeology and the Seas, 400 BCE - 1600 CE ; Université Nationale, Singapour, 23-24 avril 2019}

\section{Daniel Perret}

\section{(2) OpenEdition Journals}

Édition électronique

URL : http://journals.openedition.org/archipel/1262

DOI : 10.4000/archipel.1262

ISSN : 2104-3655

Éditeur

Association Archipel

\section{Édition imprimée}

Date de publication : 3 décembre 2019

Pagination : 3-10

ISBN : 978-2-910513-82-5

ISSN : 0044-8613

Référence électronique

Daniel Perret, « Singapura before Raffles : Archaeology and the Seas, 400 BCE - 1600 CE ; Université Nationale, Singapour, 23-24 avril 2019 », Archipel [En ligne], 98 | 2019, mis en ligne le 11 décembre 2019, consulté le 18 mars 2021. URL : http://journals.openedition.org/archipel/1262 ; DOI : https:// doi.org/10.4000/archipel.1262 


\section{ÉCHOS DE LA RECHERCHE}

\section{Singapura before Raffles: Archaeology and the Seas, 400 BCE - 1600 CE; Université Nationale, Singapour, 23- 24 avril 2019}

Onze chercheurs venus de Chine, d'Indonésie, de Malaisie, d'Allemagne, de Taiwan, de Grande-Bretagne, de Thaillande, des USA, ainsi que sept chercheurs de Singapour (NUS et Nanyang Technological University), avaient répondu à l'invitation du département des études sud-est asiatiques de l'université Nationale de Singapour afin de présenter leurs travaux lors de cette conférence organisée dans le cadre du bicentenaire de l'arrivée de Stamford Raffles à Singapour, bénéficiant ainsi du soutien financier du National Heritage Board local. L'événement, qui a réuni 180 personnes, a été ouvert par une allocution du Dr. Maliki Osman, Senior Minister of State, Ministère de la Défense et Ministère des Affaires Étrangères de Singapour.

L'idée principale de cette conférence était de mettre en lumière les avancées récentes sur la longue durée des interactions et réseaux maritimes en Asie du Sud-Est, avec un accent sur le rôle de Singapour dans ces multiples sphères de contacts, du local au global. Quatre communications étaient ainsi focalisées sur les recherches archéologiques menées à Singapour depuis les années 1980, une communication s'est intéressée à Johor, quatre communications étaient consacrées à des sites archéologiques et à des documents relatifs au détroit de Malacca, trois à la Thaïlande péninsulaire, cinq à la mer de Chine et une à la côte orientale de l'Inde, la keynote address introduisant un tableau de l'état des connaissances.

Dans sa keynote address, John Miksic (NUS) a retracé brièvement 5500 ans de connections maritimes asiatiques, en rappelant que d'après des sources cartographiques grecques, l'île de Singapour pourrait avoir été un maillon 
dans ces interactions dès le début de notre ère. Tout reste à faire en archéologie sur cette question. Les choses s'éclaircissent si l'on fait un bond en avant de 14 siècles. En effet, l'abondance des données archéologiques recueillies depuis plus de trente ans permet d'identifier alors Singapour comme un lieu de transbordement qui apparaît au tournant du XIV siècle, tout en partageant des caractéristiques communes avec ses prédécesseurs Srivijaya et Malayu. Mais une question importante demeure sans réponse: même s'il est l'héritier d'autres ports du détroit de Malacca, pourquoi Singapour émerge-t-il à ce moment-là à partir de rien?

Dans sa communication, John Miksic est revenu sur ses 35 ans de recherches archéologiques à Singapour en rappelant que tout a commencé au milieu des années 1980 avec l'initiative du Musée National de fouiller à Fort Canning. Les fouilles se sont ensuite déployées vers Parliament House Complex, les environs de l'Asian Civilisations Museum, le Singapore Cricket Club et la cathédrale Saint Andrew. Ces travaux permettent aujourd'hui des comparaisons entre secteurs, une approche qui a fait l'objet de la communication de Goh Geok Yian (Nanyang Tech. Univ.). Se basant notamment sur la nature des trouvailles faites lors des fouilles de la cathédrale Saint Andrew (20032004) et de Fort Canning Spice Gardens (2010), elle propose de distinguer des ateliers consacrés au travail du métal et du verre, un marché, une voie d'accès, et des zones de rejet. Un autre type d'étude comparative a été présenté par Foo Su Ling (NUS) qui s'est intéressée à une catégorie de porcelaine, à savoir les tessons bleu-et-blanc retrouvés dans les fouilles de Fort Canning Hill et des abords de la rivière de Singapour. L'identification des formes, des décors et des tailles des pièces remontées ou supposées lui permet de suggérer des hypothèses sur le statut social des habitants des secteurs concernés. Ces hypothèses initiales semblent toutefois contredites par les résultats de fouilles plus récentes. C'est dans le cadre d'une thèse de doctorat qu'Alasdair Chi Xin Ren (Nanyang Tech. Univ.) se consacre quant à lui à une étude en laboratoire sur des grès trouvés à Singapour et sur le site de Kota Cina (Sumatra-Nord). Sa communication a porté essentiellement sur des résultats préliminaires d'analyses de tessons de jarres dites «mercury jars». Recherchant des traces de mercure par spectrométrie de fluorescence $\mathrm{X}(\mathrm{XRF})$, il confirme des résultats obtenus préalablement sur d'autres corpus du même type de récipient, à savoir qu'il n'a détecté aucune présence de mercure à l'intérieur de ces jarres dont la fonction reste énigmatique (peut-être jarres à vin). Alasdair Chi Xin Ren a également présenté ses résultats préliminaires d'analyse physico-chimique par microscopie électronique à balayage (SEM) sur un ensemble plus varié de tessons de grès provenant de Singapour et de Kota Cina, qui indiqueraient des corpus de pâte différents.

L'hypothèse de jarre à mercure, lancée il y a près de 50 ans par F.E. Treloar suite à l'étude de pièces du musée de Sarawak à Kuching, est donc régulièrement 
invalidée depuis. Faudra-t-il attendre encore un demi-siècle avant de pouvoir constater la disparition de cette appellation erronée ?

Dans sa communication, Kwa Chong Guan (Nanyang Tech. Univ.) a posé la question des interactions entre Singapour et la rivière Johor aux $\mathrm{XVI}^{\mathrm{e}}$ et $\mathrm{XVII}^{\mathrm{e}}$ siècles. Si jusqu'à présent le bassin de la rivière Johor a livré des vestiges remontant jusqu'au milieu du XV siècle (tombes de Sayong Pinang, tessons de céramiques chinoises et de poteries, perles de Johor Lama), les seuls vestiges connus de cette époque à Singapore se limitent à quelques tessons de céramiques chinoises bleu-et-blanc retrouvés par chance dans l'estuaire de la rivière Kallang au début des années 1970. Pourtant la carte d'Eredia, datée du début du XVII ${ }^{\mathrm{e}}$ siècle, indique une activité sur l'île. Kwa Chong Guan en conclut qu'il reste un vide à combler sur le plan archéologique à Singapour pour cette période.

C'est justement Eredia et des documents du début du XVII ${ }^{\mathrm{e}}$ siècle qui ont fait l'objet de la communication de Peter Borschberg (NUS). À partir d'éléments tirés de l'autobiographie d'Eredia, de trois cartes provenant d'une collection aujourd'hui perdue et de croquis tirés de Atlas Miscelânea, Borschberg a notamment attiré l'attention sur 1'existence de multiples routes terrestres sillonnant la péninsule malaise et Sumatra à l'époque. Généralement négligées par les historiens, ces voies de communication témoignent d'une activité économique qui n'était pas limitée à la mer et aux rivières. Ces documents permettent aussi de s'interroger sur la manière dont Singapour et son espace maritime étaient connectés à ces réseaux.

Sonny Wibisono (Pusat Penelitian Arkeologi Nasional Indonesia, Jakarta) s'intéresse à cette activité économique sous l'angle des épices. Sa communication a porté sur les recherches en cours dans l'arrière-pays de Banten (Java Ouest) et à Bangka-Belitung pour retrouver les traces liées à 1'histoire de la culture et du commerce du poivre, deux étapes d'un projet dont le but est d'élaborer un modèle de la route des épices dans l'Archipel.

Plus au nord dans le détroit de Malacca, Mokhtar Saidin (Universiti Sains Malaysia, Penang) a présenté les recherches archéologiques en cours à Kedah, en particulier dans la vallée de la Bujang. Des travaux de télédétection ont permis de reconstruire l'environnement des bassins des rivières Bujang et Muda depuis le début de notre ère, confirmant les travaux antérieurs de Jane Allen sur l'existence d'une baie beaucoup plus marquée à haute époque. Ce travail préliminaire a conduit à focaliser les recherches archéologiques sur le site de Sungai Batu depuis la fin des années 2000. Une prospection géophysique a permis d'y détecter 90 monticules, dont une cinquantaine ont été fouillés à ce jour. Les nombreuses structures en briques sont interprétées comme des monuments religieux (datés à partir du $\mathrm{VI}^{\mathrm{e}}$ siècle $\mathrm{AE}$ ), des bâtiments administratifs (datés à partir du V'e siècle $\mathrm{AE}$ ), ainsi qu'un « quai » en bord de rivière $\left(\mathrm{III}^{\mathrm{e}} \mathrm{s}\right.$. EC). Un autre aspect important du complexe de Sungai 
Batu est ce que Mokhtar Saidin interprète comme des ateliers de réduction $\mathrm{du}$ fer, à partir de la découverte de fourneaux, tuyères, scories et lingots. Une datation obtenue en début d'année indiquerait une activité de réduction du fer à Sungai Batu depuis le $\mathrm{VI}^{\mathrm{e}}$ siècle $\mathrm{AE}$. La fouille d'une épave (datée également $\mathrm{du}$ VIème $\mathrm{s}$. $\mathrm{AE}$, à partir de balle de riz trouvée à l'intérieur) est en cours. Mokhtar Saidin suggère ainsi que le complexe de Sungai Batu, vestige d'une entité politique inconnue, aurait émergé au cours du $\mathrm{VI}^{\mathrm{e}}$ siècle $\mathrm{AE}$, basant sa prospérité sur l'industrie du fer.

Mokhtar Saidin n'y a pas fait allusion dans sa communication, mais ces structures et ces datations hautes ne sont pas sans soulever des interrogations majeures si on les replace dans le cadre sud-est asiatique.

Jusqu'à présent, c'est le site d'Oc-èo dans le delta du Mekong, qui aurait livré les vestiges de structures en briques les plus anciennes d'Asie du Sud-Est, peut-être datées du V $V^{e}$ siècle EC. Par ailleurs, en Asie du Sud-Est continentale, la transition entre l'Âge du bronze récent et l'Âge du fer ancien est pour l'instant datée de la seconde moitié du Ve siècle AE, alors que l'Age du métal débuterait dans l'Archipel vers 200 AE. Des vestiges de fourneaux de réduction du fer ont été retrouvés dans la région de Tagaung au Myanmar, mais l'absence de contexte stratigraphique empêche toute datation précise. Des archéologues birmans suggèrent néanmoins que Tagaung pourrait être daté entre 700 et $300 \mathrm{AE}$. On est en terrain plus sûr à Sri Ksetra, toujours au Myanmar, un site daté de la seconde moitié du $1^{\text {er }}$ millénaire EC, qui a livré de nombreux vestiges de fourneaux. Quant aux épaves retrouvées en Asie du Sud-Est, la plus ancienne avérée à ce jour serait celle de Pontian, en Péninsule malaise (voir toutefois la communication d'Amara Srisuchat ci-dessous). Elle est datée entre la fin du $\mathrm{III}^{\mathrm{e}}$ et le début du $\mathrm{V}^{\mathrm{e}}$ siècle $\mathrm{EC}^{1}$. Le complexe de Sungai Batu abriterait par conséquent les plus anciennes structures en briques connues en Asie du Sud-Est (un bond en arrière d'un millénaire), les vestiges les plus anciens de fours à réduction du fer (peut-être un bond en arrière de plus d'un millénaire) et l'épave la plus ancienne (un bond en arrière d'au moins quatre siècles). Il y a donc tout lieu d'être prudent sur ces datations en attendant une publication détaillée des résultats de ces fouilles de grande envergure menées à Sungai Batu. La communication de Mokhtar Saidin amène trois observations supplémentaires. L'interprétation d'une structure en briques comme un quai en bord de rivière est très probablement unique à ce jour en Asie du Sud-Est pour les premiers siècles de notre ère. Si elle est validée, il faudrait se tourner vers l'Inde pour y chercher des structures similaires à la même époque. Par ailleurs, sur les images présentées les fourneaux à réduction de fer apparaissent intacts, alors qu'en principe ils doivent être cassés à la fin de chaque opération de réduction. S'il s'agit bien de fourneaux, leur état suggérerait que le site a

1. John Miksic \& Geok Yian Goh, Ancient Southeast Asia, London, New York, Routledge, 2017, pp. 104, 106, 162, 203, 212, 273-274. 
été abandonné précipitamment pour une raison inconnue. Enfin, à part des briques et des vestiges liés à l'industrie de fer, Mokhtar Saidin n'a évoqué aucune autre trouvaille de mobilier, en particulier des poteries.

La communication de Daniel Perret (EFEO) a porté sur des résultats préliminaires de fouilles menées entre 2011 et 2016 sur le site d'habitat ancien de Kota Cina, près de Medan à Sumatra, en coopération avec le Centre national de la recherche archéologique d'Indonésie. L'abondance des données et $\mathrm{du}$ mobilier (céramiques chinoises, poteries, vestiges de faune, etc.) recueillis suite à la fouille de près de 70 sondages devrait permettre de préciser l'histoire de ce site d'habitat redécouvert il y a près de 50 ans, de repenser ses interactions avec des sites contemporains de la région, ainsi que son rôle dans les réseaux reliant l'océan Indien à la mer de Chine. Son abandon serait contemporain de l'émergence de Singapour.

La Thaïlande péninsulaire a fait l'objet de trois communications. Aude Favereau (National Cheng Kung Univ., Taiwan) a présenté les résultats d'une étude technologique sur des corpus de poteries originaires de trois sites de la côte orientale de la péninsule datés entre le $\mathrm{IV}^{\mathrm{e}}$ siècle $\mathrm{AE}$ et les tous premiers siècles de notre ère: Khao Sam Kaeo, Khao Sek et Tha Chana. Cette étude a permis de préciser la répartition des types entre ces trois sites, de mettre en lumière l'usage de technologies et styles étrangers (en particulier indiens), et d'identifier des importations (Golfe du Bengale et mer de Chine méridionale). Si Khao Sek apparaît alors comme un site mineur, Khao Sam Kaeo et Tha Chana ont abrité des communautés étrangères.

Amara Srisuchat (Dept. of Fine Arts, Thaïlande) a livré un tableau des découvertes archéologiques et des réinterprétations de sources anciennes faites durant ces dernières décennies, surtout à propos de la Thaïlande péninsulaire, découvertes datées entre $400 \mathrm{AE}$ et 1600 EC. Six catégories de trouvailles ont été abordées: épaves, tambours de bronze et lingots, poteries et céramiques, perles, monnaies, tablettes votives et stupas miniatures. En ce qui concerne les épaves, il faut signaler la découverte d'un bateau à voile avec une datation proposée de ca. $200 \mathrm{AE}$ (datation qui situerait cette épave entre celles de Sungai Batu et de Pontian en Malaisie, signalées plus haut), ainsi que d'un boutre (bateau traditionnel de la mer Rouge) daté du IX ${ }^{\mathrm{e}}$ siècle EC. Mentionnons également la découverte sur le site de Si Thep d'une tablette votive bilingue (sanskrit/chinois) datée du VIII ${ }^{\mathrm{e}}$ siècle EC.

L'entité politique de Tambralinga a fait l'objet de la communication de Wannasarn Noonsuk (California State University, Fresno). Tambralinga émerge durant les premiers siècles de notre ère et devient un royaume côtier important au $\mathrm{V}^{\mathrm{e}}-\mathrm{VI}^{\mathrm{e}}$ siècles. Cette entité, connectée aux réseaux maritimes, en particulier à Srivijaya, regroupait des communautés implantées le long de plusieurs rivières courtes reliant les montagnes de l'ouest à la côte orientale. Par ailleurs, d'anciennes lignes de plages permettaient à ces communautés de 
communiquer du nord au sud. Des recherches récentes menées sur le Grand Stupa de Nakhon montrent des liens artistiques avec l'art de Java et l'art Pala aux IX $-\mathrm{X}^{\mathrm{e}}$ siècles. L'entité semble faire place au royaume de Nakhon $\mathrm{Si}$ Thammarat au XIII ${ }^{e}$ siècle, qui devient notamment un centre de production de poteries fines (Fine Paste Wares).

Dans sa communication, Michael Flecker (Maritime Explorations, Singapore) a comparé les cargaisons de trois épaves datées du XII siècle : celle du Pulau Buaya Wreck récupérée en 1989, et plus récemment celles du Lingga Wreck, qui a coulé non loin de Pulau Buaya, et celle du Flying Fish Wreck (publication à paraître) retrouvée au large de Kota Kinabalu (Sabah). Tous trois étaient des bateaux sud-est asiatiques transportant des céramiques chinoises et du fer en barres vers des marchés d'Asie du Sud-Est. Si les cargaisons des deux dernières ont peu de types de céramiques en commun, celle du Pulau Buaya Wreck a livré de nombreux types communs avec chacune d'elles. Ces comparaisons permettent de dater ces trois épaves du premier quart du XII ${ }^{e}$ siècle et de les qualifier de «sister ships ».

Naniek Harkantiningsih (Pusat Penelitian Arkeologi Nasional Indonesia, Jakarta) a présenté une communication sur ses recherches menées depuis 2010 en mer de Chine méridionale, dans l'archipel de Natuna composé de plus de 150 îles. Elles ont révélé l'existence de très nombreuses tombes accompagnées de céramiques chinoises, mobilier qui met en lumière le rôle de l'archipel dans l'histoire des routes maritimes anciennes en mer de Chine. Ces tombes à faible profondeur et situées sur des plages font malheureusement l'objet d'un pillage systématique.

La communication de Sun Jian (National Centre of Underwater Cultural Heritage, China) a porté sur la fouille et l'étude en cours de l'épave du Nanhai I, localisée à la fin des années 1980 sur les côtes de la province du Guangdong. Cette épave ( $22 \mathrm{~m}$ x 9,9 m) a fait l'objet d'une opération spectaculaire, puisqu'elle a été remontée en un bloc à la surface avant d'être transportée au musée de la Route maritime de la soie du Guangdong pour y être fouillée systématiquement. Datée de la fin du XII ${ }^{\mathrm{e}}$ siècle, sa cargaison (160 000 pièces pour un poids de 400-500 tonnes) comprend principalement des céramiques chinoises, du fer en barres (120 tonnes), du cuivre et des monnaies à destination de l'Asie du Sud-Est. L'épave a livré également des données importantes concernant la vie quotidienne sur le bateau. L'état de conservation exceptionnel permet d'en étudier la structure ainsi que le système de stockage de la cargaison en plusieurs niveaux. Un volume (en chinois) sur la fouille a déjà été publié, d'autres suivront.

Xin Guangcan (National Centre of Underwater Cultural Heritage, China) a présenté une communication centrée sur le XIV e siècle, dans laquelle sont comparées les céramiques chinoises trouvées à Singapour avec des pièces retrouvées dans les fouilles d'entrepôts contemporains de Quanzhou et de Taicang, près de l'actuelle Shanghai. 
Cai Yunci (Leicester Univ., UK) a présenté dans sa communication l'expérience du port de Quanzhou (Fujian). Dans le contexte du projet Belt and Road Initiative (BRI), le gouvernement chinois a présenté en juillet 2018 la candidature de la ville à l'inscription sur la liste du patrimoine mondial de l'UNESCO, mettant en avant le rôle du port dans les échanges entre Chinois et étrangers durant la période $\mathrm{X}^{\mathrm{e}}-\mathrm{XIV}^{\mathrm{e}}$ siècles. Cao Yunci s'est intéressée à la fois aux effets collatéraux de cette candidature dans la ville de Quanzhou même (gentrification, désertion de monuments soumis à des réglements drastiques, développement de pélerinages sur des tombes musulmanes mises en valeur, etc.), ainsi qu'aux raisons de son échec qui s'expliquerait avant tout par des systèmes de valeurs différents en Occident et en Asie.

L'océan Indien a été abordé par Himanshu Prabha Ray (Ludwig Maximilian Univ., Munich) avec une communication traitant des sites à poterie roulettée le long de la côte orientale de l'Inde. Elle a d'abord rappelé qu'il est maintenant largement accepté que ce type de poterie fait son apparition durant les $\mathrm{III}^{\mathrm{e}}-\mathrm{II}{ }^{\mathrm{e}}$ siècles AE et qu'elle est présente du Bengale au Sri Lanka, ainsi qu'en Asie du Sud-Est (Vietnam, Thaïlande, Malaisie, Indonésie). À partir des données archéologiques disponibles à propos de la côte orientale de l'Inde, Himanshu Prabha Ray s'est ici interrogée sur la ou les fonctions de cette poterie, l'organisation de son commerce, ainsi que sur sa présence en Asie du Sud-Est. Elle relève notamment son absence sur la côte occidentale de l'Inde et propose des pistes de recherche telles sa présence sur les plus anciens sites bouddhiques ou encore son apparition contemporaine du nigama, la villemarché, sur la côte orientale de l'Inde.

$\mathrm{Au}$ final, une conférence stimulante et fructueuse qui a permis de partager des résultats et des réflexions sur des recherches en cours dans divers domaines s'éclairant mutuellement avec la présentation de nombreuses données inédites. Au-delà, elle a aussi illustré le fait que l'accumulation des données archéologiques acquises dans des conditions satisfaisantes depuis plusieurs décennies offre maintenant des bases solides pour préciser des datations et établir des comparaisons de natures diverses qui ne peuvent qu'enrichir les connaissances sur l'histoire ancienne de l'Asie du Sud-Est.

DANIEL PERRET ${ }^{2}$

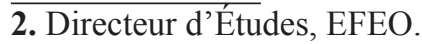




\section{Liste des communications (dans l'ordre de présentation)}

Keynote address:

John Miksic. Connecting the Dots: Nodes and Networks in Early Asian Maritime Trade Communications:

- Himanshu Prabha Ray. Distribution of Rouletted Ware Sites along the East Coast of India and the Organisation of Maritime Trade

- Aude Favereau. Late Prehistoric Ports in Peninsular Thailand: Investigating Local and Long Distance Connections through Pottery

- Cai Yunci. Building Sustainable Futures through our Collective Maritime Heritage: The Nomination of Quanzhou on the UNESCO World Heritage List and its Lessons for Singapore

- Michael Flecker. Sister Ships: Three 12th Century Shipwrecks in Southeast Asia

- Kwa Chong Guan. The Archaeology of 16th-17th Century Johor River and its Relevance to Singapore Towards a Connected History

- Peter Borschberg. Observations on Three Maps Ascribed to Manuel Godinho de Eredia

- John Miksic. 35 Years of Archaeological Research in Singapore

- Goh Geok Yian. A Tale of Two Sites: Temasek in the Asian Maritime System

- Alasdair Chi Xin Ren. Trade and Exchange along the Maritime Silk Route: High-Resolution Analysis of "Mercury Jars" and other Stoneware from Early Singapore and Kota Cina, Sumatra

- Foo Su Ling. On the Hill and By the River: Blue and White Ceramic Sherds from 14th Century Singapura

- Sun Jian. New Sights on the Nanhai Shipwreck

- Xin Guanggan. A Preliminary Study on Several 14th Century Port Cities from China to Southeast Asia

- Mokhtar Saidin. Kedah Tua since the 6th century BCE

- Amara Srisuchat. Trace of Maritime Contacts: Thailand Significant Sites and Findings

- Wannasarn Noonsuk. Tambralinga, Srivijaya and Maritime Southeast Asia: Recent Discoveries

- Sonny Wibisono. The Production Chain in the Spice Commerce Network: A Review of Archaeological Research in the Western Region of the Archipelago

- Daniel Perret. The Kota Cina Settlement in North Sumatra: French-Indonesia Excavations (2011-2016)

- Naniek Harkantiningsih. Natuna as a Trajectory of Cultural and Commercial Global Networks 